\title{
Guest editorial: social media for personalization and search
}

\author{
Ludovico Boratto $^{1} \cdot$ Andreas Kaltenbrunner ${ }^{2,3} \cdot$ Giovanni Stilo $^{4}$
}

Published online: 21 February 2019

(c) Springer Nature B.V. 2019

\section{Introduction}

Social media platforms have become powerful tools to collect the preferences of the users and get to know them more. In order to build profiles about what the users like or dislike, a system does not only have to rely on explicitly given preferences (e.g., ratings) or on implicitly collected data (e.g., from the browsing sessions). In the middle, there lie opinions and preferences expressed through likes, textual comments, click/view logs, following preferences, digital conversations, and posted content. Moreover, the social network itself can provide information on who influences whom.

In order to improve the web experience of the users, classic personalization technologies (e.g., recommender systems) and search engines usually rely on static schemes. Users are allowed to express ratings in a fixed range of values for a given catalog of products, or to express a query that usually returns the same set of webpages/products for all the users.

Being able to mine usage and collaboration patterns in social media and to analyze the content generated by the users opens new frontiers in the generation of personalization services and in the improvement of search engines. Moreover, recent technological advances, such as deep learning, are able to provide a context to the analyzed data.

In this special issue, we explore how social media can be employed for personalization and search purposes. The rest of this article is structured as follows: in Sect. 2 we introduce some recent advances in this area and Sect. 3 contains concluding remarks.

Ludovico Boratto

ludovico.boratto@acm.org

Andreas Kaltenbrunner

kaltenbrunner@gmail.com

Giovanni Stilo

giovanni.stilo@univaq.it

1 Eurecat, Centre Tecnológic de Catalunya, Barcelona, Spain

2 NTENT, Barcelona, Spain

3 Universitat Pompeu Fabra, Barcelona, Spain

4 University of L'Aquila, L'Aquila, Italy 


\section{In this special issue}

The seven accepted articles in this special issue propose innovative techniques that use information coming from social media to provide personalization and improve search results. The research contributions advance the state of the art, considering different aspects and scenarios, ranging from content and user recommendation, to search bias quantification, by employing different social media platforms, such as Twitter and Wikipedia.

In their article "A topic recommender for journalists", Cucchiarelli et al. (2018) present a recommender system, named What to Write and Why, that suggests to journalists topics that are possibly interesting for the users but that have not been sufficiently covered in news articles. The information about these topics comes from an analysis of Twitter and Wikipedia data, and the methodology proposed by the authors temporally aligns the information collected from these two sources with the news articles. The effectiveness of the approach is validated both with offline experiments that aim at capturing the saliency and serendipity of the recommendations, and with a manual evaluation performed by experts.

Monteserin and Armentano (2018) considered the role of social influence to link users, in the paper "Influence me! Predicting links to influential users". The proposed recommender system infers the social influence of the users in a social network. This is done thanks to a data-based approach named Credit Distribution, which learns how influence flows in a network by directly leveraging available propagation traces. Influential users are then recommended to a target user, to overcome issues that occur in case of low homophily.

Eberhard et al. (2018) in their article "Predicting trading interactions in an online marketplace through location-based and online social networks", investigate the extent to which trading interactions between sellers and buyers within an online marketplace platform can be predicted. They do so considering three different but overlapping networks, i.e., an online social network, a location-based social network and a trading network. Experiments are performed in the context of the virtual world Second Life, where the authors crawled data of the online social network, user information of the location-based social network, and purchases of the trading network.

The paper "User interest prediction over future unobserved topics on social networks", by Zarrinkalam et al. (2018), aims at predicting the future interests of the users. To do so, the authors propose a framework that monitors the temporal evolution of the user preferences and utilizes semantic information from knowledge bases such as Wikipedia to make the prediction. The framework is evaluated and validated thanks to a comparison with other state-of-the-art approaches on a real-world Twitter dataset.

In their article "Determining the interests of social media users: two approaches", Seghouani et al. (2018) propose two ways to determine the interests of social media users. Characterizing user interests can be helpful to recommend the users services, similar people, or to predict future interests. The first approach, named Frisk, is an unsupervised multilingual approach that considers the explicit meaning of the words that occur in the users posts. The second, named Ascertain, is a supervised approach that resorts to the hidden dimensions of the words, in order to reveal some of the psychological processes and personality traits of a person. Experiments are performed on Twitter data, to validate the capability of the approaches to infer the interests in a multilingual context and to exploit psychological traits in an effective way.

Naini et al. (2018) in their article "Those were the days: learning to rank social media posts for reminiscence", aim at understanding which posts should be considered as memorable, in order to effectively summarize the previous social activities of a user. The authors first perform user evaluation studies and construct a corpus that captures human expectations towards 
content retention. After these studies, they select a set of features that are most likely to characterize memorable posts and propose machine-learning models to rank posts for retention.

The final paper of this special issue, titled "Search bias quantification: investigating political bias in social media and web search", by Kulshrestha et al. (2018), presents a bias quantification framework that measures the political bias in ranked list output by the search system. Moreover, it also decouples the bias introduced by the different sources, i.e., the input data and and the ranking system. The framework is validated both on a specific use case (the 2016 US Presidential primaries in Twitter social media search) and to compare the relative bias for two popular search systems (Twitter social media search and Google web search).

\section{Conclusions}

Extracting information from social media is a topic of central interest to gather actionable knowledge about the users and provide effective personalization and search results to them. The papers included in this special issue cover several topics and present some of the key directions in this vibrant and rapidly expanding area of research and development. We hope the set of selected papers provides the community with a better understanding of the current directions, and that they inspire readers with possible areas to focus on in their future research.

Acknowledgements We thank all the authors for considering this special issue as an outlet to publish their research results in the area of social media for personalization and search. We also would like to thank the referees who provided very useful and thoughtful feedback to the authors. Finally, we express our gratitude to the Editors-in-Chief, for their kind support, advice, and encouragements throughout the preparation of this special issue.

\section{References}

Cucchiarelli, A., Morbidoni, C., Stilo, G., \& Velardi, P. (2018). A topic recommender for journalists. Information Retrieval Journal. https://doi.org/10.1007/s10791-018-9333-2.

Eberhard, L., Trattner, C., \& Atzmueller, M. (2018). Predicting trading interactions in an online marketplace through location-based and online social networks. Information Retrieval Journal. https://doi.org/10.1007/ s10791-018-9336-Z.

Kulshrestha, J., Eslami, M., Messias, J., Zafar, M. B., Ghosh, S., Gummadi, K. P., et al. (2018). Search bias quantification: Investigating political bias in social media and web search. Information Retrieval Journal. https://doi.org/10.1007/s10791-018-9341-2.

Monteserin, A., \& Armentano, M. G. (2018). Influence me! predicting links to influential users. Information Retrieval Journal. https://doi.org/10.1007/s10791-018-9335-0.

Naini, K. D., Kawase, R., Kanhabua, N., Niederée, C., \& Altingovde, I. S. (2018). Those were the days: Learning to rank social media posts for reminiscence. Information Retrieval Journal. https://doi.org/10.1007/ s10791-018-9339-9.

Seghouani, N. B., Jipmo, C. N., \& Quercini, G. (2018). Determining the interests of social media users: Two approaches. Information Retrieval Journal. https://doi.org/10.1007/s10791-018-9338-X.

Zarrinkalam, F., Kahani, M., \& Bagheri, E. (2018). User interest prediction over future unobserved topics on social networks. Information Retrieval Journal. https://doi.org/10.1007/s10791-018-9337-y.

Publisher's Note Springer Nature remains neutral with regard to jurisdictional claims in published maps and institutional affiliations. 